\title{
On pgra Homeomorphisms in Intuitionistic Fuzzy Topological Spaces
}

\author{
M. Thirumalaiswamy \\ Department of Mathematics, \\ NGM college, Pollachi - 642 001, \\ Tamil Nadu, India.
}

\author{
A. Surya \\ Department of Mathematics, \\ PA Polytechnic College, Pollachi- 642 002, \\ Tamil Nadu, India.
} Abstract: In this paper, we introduce intuitionistic fuzzy pgr $\alpha$ closed mapping, intuitionistic fuzzy pgr $\alpha$ open mapping,
intuitionistic fuzzy pgr $\alpha$ homeomorphisms and study some of their properties in intuitionistic fuzzy topological spaces.

Keywords and Phrases: Intuitionistic fuzzy topology, intuitionistic fuzzy set, intuitionistic fuzzy pgr $\alpha$ continuous, intuitionistic fuzzy pgr $\alpha$ open mapping, intuitionistic fuzzy pgr $\alpha$ closed mapping and intuitionistic fuzzy pgr $\alpha$ homeomorphisms.

\section{Mathematics Subject Classification: 54A40, 03F55}

\section{INTRODUCTION}

The concept of fuzzy sets was introduced by Zadeh [16] and later Atanassov [1] generalized this idea to intuitionistic fuzzy sets by using the notation of fuzzy sets. On the other hand Coker [2] introduced intuitionistic fuzzy topological spaces using the notion of intuitionistic fuzzy sets. In this paper, we introduce intuitionistic fuzzy pgr $\alpha$ closed mapping, intuitionistic fuzzy pgr $\alpha$ open mapping, intuitionistic fuzzy pgr $\alpha$ homeomorphisms and study some of their properties.

\section{PRELIMINARIES}

Throughout this paper, $(\mathrm{X}, \tau),(\mathrm{Y}, \sigma)$ and $(\mathrm{Z}, \gamma)$ (or simply $\mathrm{X}$, $\mathrm{Y}$ and $\mathrm{Z}$ ) denote the intuitionistic fuzzy topological spaces (IFTS for short) on which no separation axioms are assumed unless otherwise explicitly mentioned. For a subset $\mathrm{A}$ of $\mathrm{X}$, the closure, the interior and the complement of $\mathrm{A}$ are denoted by $\mathrm{cl}(\mathrm{A})$, int( $\mathrm{A})$ and $\mathrm{A}^{\mathrm{c}}$ respectively. We recall some basic definitions that are used in the sequal.

\subsection{Definition [1]}

Let $\mathrm{X}$ be a nonempty set. An intuitionistic fuzzy set (IFS for short) $\mathrm{A}$ in $\mathrm{X}$ is an object having the form $\mathrm{A}=\left\langle x, \mu_{A}, v_{A} ; x \in X\right\rangle$ where the functions $\mu_{A}: \mathrm{X} \rightarrow[0,1]$ and $v_{A}: \mathrm{X} \rightarrow[0,1]$ denote the degree of membership (namely $\mu_{A}(x)$ ) and the degree of non membership (namely $v_{A}(x)$ ) of each element $x \in X$ to the set A, respectively, and $0 \leq \mu_{A}(x)+v_{A}(x) \leq 1$ for each $x \in X$. Denote by $\operatorname{IFS}(\mathrm{X})$, the set of all intuitionistic fuzzy sets in $X$.

\subsection{Definition [1]}

Let $\mathrm{A}$ and $\mathrm{B}$ be IFSs of the form $\mathrm{A}=$

$\left\{\left\langle x, \mu_{A}(x), v_{A}(x)\right\rangle: x \in X\right\}$ and $\mathrm{B}=\left\{\left\langle x, \mu_{B}(x), v_{B}(x)\right\rangle: x \in\right.$ $X\}$. Then

1. A $\subseteq \mathrm{B}$ if and only if $\mu_{A}(x) \leq \mu_{B}(x)$ and $v_{A}(x) \geq v_{B}(x)$

for all $x \in X$,

2. $\mathrm{A}=\mathrm{B}$ if and only if $\mathrm{A} \subseteq \mathrm{B}$ and $\mathrm{B} \subseteq A$,

3. $\mathrm{A}^{\mathrm{c}}=\left\{\left\langle x, v_{A}(x), \mu_{A}(x)\right\rangle: x \in X\right\}$,

4. $\mathrm{A} \cap \mathrm{B}=\left\{\left\langle x, \mu_{A}(x) \wedge \mu_{B}(x), v_{A}(x) \vee v_{B}(x)\right\rangle: x \in X\right\}$,

$$
\text { 5. } \mathrm{A} \cup \mathrm{B}=\left\{\left\langle x, \mu_{A}(x) \vee \mu_{B}(x), v_{A}(x) \wedge v_{B}(x)\right\rangle: x \in X\right\} .
$$

For the sake of simplicity, we shall use the notation $\mathrm{A}=\left\langle x, \mu_{A}, v_{A}\right\rangle$ instead of $\mathrm{A}=\left\{\left\langle x, \mu_{A}(x), v_{A}(x)\right\rangle ; x \in X\right\}$. The intuitionistic fuzzy sets $0_{\sim}=\{\langle x, 0,1\rangle: x \in X\}$ and $1_{\sim}=$ $\{\langle x, 1,0\rangle: x \in X\}$ are respectively the empty set and the whole set of $X$.

\subsection{Definition [2]}

An intuitionistic fuzzy topology (IFT for short) on $\mathrm{X}$ is a family $\tau$ of IFSs in X satisfying the following axioms.

1. $0_{\sim}, 1_{\sim} \in \tau$,

2. $G_{1} \cap G_{2} \in \tau$ for any $G_{1}, G_{2} \in \tau$,

3. $\cup G_{i} \in \tau$ for any family $\left\{G_{i}: i \in J\right\} \subseteq \tau$.

In this case the pair $(\mathrm{X}, \tau)$ is called an intuitionistic fuzzy topological space (IFTS for short) and any IFS in $\tau$ is known as an intuitionistic fuzzy open set (IFOS for short) in X. The complement $\mathrm{A}^{\mathrm{c}}$ of an IFOS A in an IFTS $(\mathrm{X}, \tau)$ is called an intuitionistic fuzzy closed set (IFCS for short) in $\mathrm{X}$.

\subsection{Definition [2]}

Let $(\mathrm{X}, \tau)$ be an IFTS and $\mathrm{A}=\left\langle x, \mu_{A}, v_{A}\right\rangle$ be an IFS in $\mathrm{X}$. Then

1. $\operatorname{int}(\mathrm{A})=\cup\{G: G$ is an IFOS in $\mathrm{X}$ and $\mathrm{G} \subseteq A\}$,

2. $\operatorname{cl}(\mathrm{A})=\cap\{\mathrm{K}: \mathrm{K}$ is an IFCS in $\mathrm{X}$ and $\mathrm{A} \subseteq K\}$.

For any IFS $\mathrm{A}$ in $(\mathrm{X}, \tau)$, we have $\mathrm{cl}\left(\mathrm{A}^{\mathrm{c}}\right)=(\operatorname{int}(\mathrm{A}))^{\mathrm{c}}$ and $\operatorname{int}\left(\mathrm{A}^{\mathrm{c}}\right)=(\operatorname{cl}(\mathrm{A}))^{\mathrm{c}}$

\subsection{Definition [3]}

An IFS $\mathrm{A}=\left\langle x, \mu_{A}, v_{A}\right\rangle$ in an IFTS $(\mathrm{X}, \tau)$ is said to be an 1. intuitionistic fuzzy semi closed set (IFSCS for short) if $\operatorname{int}(\operatorname{cl}(\mathrm{A})) \subseteq A$,

2. intuitionistic fuzzy semi open set (IFSOS for short) if $A \subseteq \operatorname{cl}(\operatorname{int}((\mathrm{A}))$

3. intuitionistic fuzzy pre closed set (IFPCS for short) if $\operatorname{cl}(\operatorname{int}((\mathrm{A})) \subseteq A$, 
4. intuitionistic fuzzy pre open set (IFPOS for short) if $A \subseteq \operatorname{int}(\operatorname{cl}(\mathrm{A}))$,

5. intuitionistic fuzzy regular closed set (IFRCS for short) if $\operatorname{cl}(\operatorname{int}(\mathrm{A}))=A$,

6. intuitionistic fuzzy regular open set (IFROS for short) if $\mathrm{A}=\operatorname{int}(\mathrm{cl}(\mathrm{A}))$,

7. intuitionistic fuzzy $\alpha$ closed set (IF $\alpha C \mathrm{~S}$ for short) if $\operatorname{cl}(\operatorname{int}(\operatorname{cl}(\mathrm{A}))) \subseteq A$,

8. intuitionistic fuzzy $\alpha$ open set (IF $\alpha O \mathrm{~S}$ for short) if $\mathrm{A} \subseteq$ $\operatorname{int}(\operatorname{cl}(\operatorname{int}(\mathrm{A})))$.

\subsection{Definition}

Let $\mathrm{A}=\left\langle x, \mu_{A}, v_{A}\right\rangle$ be an IFS in an IFTS $(\mathrm{X}, \tau)$. Then 1. $\alpha \operatorname{int}(\mathrm{A})=\cup\{G: G$ is an IF $\alpha \mathrm{OS}$ in $\mathrm{X}$ and $\mathrm{G} \subseteq A\}$ and $\alpha \operatorname{cl}(\mathrm{A})=\cap\{\mathrm{K}: \mathrm{K}$ is an IF $\alpha \mathrm{CS}$ in $\mathrm{X}$ and $\mathrm{A} \subseteq K\}[9]$, 2. $p \operatorname{int}(\mathrm{A}=\cup\{G: G$ is an IFPOS in $\mathrm{X}$ and $\mathrm{G} \subseteq A\}$ and $p \operatorname{cl}(\mathrm{A})=\cap\{\mathrm{K}: \mathrm{K}$ is an IFPCS in $\mathrm{X}$ and $\mathrm{A} \subseteq K\}[3]$, 3. $\operatorname{sint}(\mathrm{A})=\cup\{G: G$ is an IFSOS in $\mathrm{X}$ and $\mathrm{G} \subseteq A\}$ and $s \mathrm{cl}(\mathrm{A})=\cap\{\mathrm{K}: \mathrm{K}$ is an IFSCS in $\mathrm{X}$ and $\mathrm{A} \subseteq K\}[9]$.

\subsection{Definition [15]}

An IFS A of an IFTS $(X, \tau)$ is called an intuitionistic fuzzy regular $\alpha$ open set ((IFR $\alpha$ OS for short) if there exist an IFROS $\mathrm{U}$ such that $\mathrm{U} \subseteq A \subseteq \alpha \mathrm{cl}(\mathrm{U})$.

\subsection{Definition}

An IFS $\mathrm{A}=\left\langle x, \mu_{A}, v_{A}\right\rangle$ in an IFTS $(\mathrm{X}, \tau)$ is called an

1. intuitionistic fuzzy generalized closed set (IFGCS for short) if $\operatorname{cl}(\mathrm{A}) \subseteq U$, whenever $\mathrm{A} \subseteq U$ and $\mathrm{U}$ is an IFOS in $\mathrm{X}[13]$,

2. intuitionistic fuzzy $\alpha$ generalized closed set (IF $\alpha$ GCS for short) if $\alpha \operatorname{cl}(\mathrm{A}) \subseteq U$, whenever $\mathrm{A} \subseteq U$ and $\mathrm{U}$ is an IFOS in $\mathrm{X}[9]$,

3. intuitionistic fuzzy weakly generalized closed set (IFWGCS for short) if $\operatorname{cl}(\operatorname{int}(\mathrm{A})) \subseteq U$, whenever $\mathrm{A} \subseteq U$ and $\mathrm{U}$ is an IFOS in $\mathrm{X}$ [7],

4. intuitionistic fuzzy generalized semi closed set (IFGSCS for short) if $\operatorname{scl}(\mathrm{A}) \subseteq U$, whenever $\mathrm{A} \subseteq U$ and $\mathrm{U}$ is an IFOS in $X$ [11],

5. intuitionistic fuzzy regular generalized $\alpha$ closed set (IFRG $\alpha \mathrm{CS}$ for short) if $\alpha \mathrm{cl}(\mathrm{A}) \subseteq U$, whenever $\mathrm{A} \subseteq U$ and $\mathrm{U}$ is an IFR $\alpha \mathrm{OS}$ in $\mathrm{X}$ [5].

An IFS A is said to be an intuitionistic fuzzy generalized open set (IFGOS for short), intuitionistic fuzzy $\alpha$ generalized open set (IF $\alpha$ GOS for short), intuitionistic fuzzy weakly generalized open set (IFWGOS for short), intuitionistic fuzzy generalized semi open set (IFGSOS for short) and intuitionistic fuzzy regular generalized $\alpha$ open set (IFRG $\alpha$ OS for short) if the complement of $\mathrm{A}$ is an IFGCS, IF $\alpha$ GCS, IFWGCS, IFGSCS, and IFRG $\alpha$ CS respectively

\subsection{Definition [6]}

An IFS $\mathrm{A}=\left\langle x, \mu_{A}, v_{A}\right\rangle$ in an IFTS $(\mathrm{X}, \tau)$ is called an intuitionistic fuzzy regular weakly generalized closed set (IFRWGCS for short) if $\operatorname{cl}(\operatorname{int}(\mathrm{A})) \subseteq U$, whenever $\mathrm{A} \subseteq \mathrm{U}$ and $U$ is an IFROS in X. An IFS A is called an intuitionistic fuzzy regular weakly generalized open set
(IFRWGOS for short) in $\mathrm{X}$ if the complement of $\mathrm{A}$ is an IFRWGCS in X.

\subsection{Definition [14]}

An IFS A in an IFTS $(X, \tau)$ is said to be an intuitionistic fuzzy pgr $\alpha$ closed set (IF pgr $\alpha C S$ for short) if pcl (A) $\subseteq \mathrm{U}$ whenever $\mathrm{A} \subseteq \mathrm{U}$ and $\mathrm{U}$ is an IFR $\alpha \mathrm{OS}$ in $\mathrm{X}$. The family of all IF pgr $\alpha$ CSs of an IFTS $(\mathrm{X}, \tau)$ is denote by IFpgr $\alpha \mathrm{C}(\mathrm{X})$.

\subsection{Definition [3]}

A mapping $\mathrm{f}:(\mathrm{X}, \tau) \rightarrow(\mathrm{Y}, \sigma)$ is called an intuitionistic fuzzy continuous mapping (IF continuous mapping for short) if $f^{-1}(\mathrm{~B})$ is an IFOS in $(\mathrm{X}, \tau)$ for every IFOS B of $(\mathrm{Y}, \sigma)$.

\subsection{Definition [4]}

Let $\mathrm{f}:(\mathrm{X}, \tau) \rightarrow(\mathrm{Y}, \sigma)$ be a mapping. Then $\mathrm{f}$ is said to be an 1. intuitionistic fuzzy $\alpha$ continuous mapping (IF $\alpha$ continuous mapping for short) if $f^{-1}(\mathrm{~B}) \epsilon \operatorname{IF} \alpha \mathrm{O}(\mathrm{X})$ for every $\mathrm{B} \epsilon \sigma$,

2. intuitionistic fuzzy pre continuous mapping (IFP continuous mapping for short) if $f^{-1}(\mathrm{~B}) \epsilon \operatorname{IFPO}(\mathrm{X})$ for every $\mathrm{B} \epsilon \sigma$.

\subsection{Defintion [5]}

Let $\mathrm{f}:(\mathrm{X}, \tau) \rightarrow(\mathrm{Y}, \sigma)$ be a mapping. Then $\mathrm{f}$ is said to be an intuitionistic fuzzy regular generalized $\alpha$ continuous mapping (IFRG $\alpha$ continuous mapping for short) if $f^{-1}(\mathrm{~B})$ is an IFRG $\alpha$ CS in $(\mathrm{X}, \tau)$ for every $\operatorname{IFCS} \mathrm{B}$ of $(\mathrm{Y}, \sigma)$.

\subsection{Definition $[8]$}

Let $\mathrm{f}:(\mathrm{X}, \tau) \rightarrow(\mathrm{Y}, \sigma)$ be a mapping. Then $\mathrm{f}$ is said to be an intuitionistic fuzzy regular weakly generalized continuous mapping (IFRWG continuous mapping for short) if $f^{-1}(\mathrm{~B})$ is an IFRWGCS in $(\mathrm{X}, \tau)$ for every $\operatorname{IFCS} \mathrm{B}$ of $(\mathrm{Y}, \sigma)$.

\subsection{Definition [14]}

A mapping $\mathrm{f}:(\mathrm{X}, \tau) \rightarrow(\mathrm{Y}, \sigma)$ is called an intuitionistic fuzzy pgr $\alpha$ continuous (IFpgr $\alpha$ continuous for short) mapping if $f^{-1}(\mathrm{~V})$ is an IFpgr $\alpha \mathrm{CS}$ in $(\mathrm{X}, \tau)$ for every IFCS V of $(\mathrm{Y}, \sigma)$.

\subsection{Definition [12]}

A mapping $\mathrm{f}:(\mathrm{X}, \tau) \rightarrow(\mathrm{Y}, \sigma)$ is called an intuitionistic fuzzy closed mapping (IFCM for short) if $\mathrm{f}(\mathrm{A})$ is an IFCS in $\mathrm{Y}$ for each IFCS A in X.

\subsection{Definition [12]}

A mapping $\mathrm{f}:(\mathrm{X}, \tau) \rightarrow(\mathrm{Y}, \sigma)$ is called an

1. intuitionistic fuzzy semi open mapping (IFSOM for short) if $\mathrm{f}(\mathrm{A})$ is an IFSOS in Y for each IFOS A in X.

2. intuitionistic fuzzy pre open mapping (IFPOM for short) if $\mathrm{f}(\mathrm{A})$ is an IFPOS in $\mathrm{Y}$ for each IFOS $\mathrm{A}$ in $\mathrm{X}$.

\subsection{Definition [5]}

A mapping $\mathrm{f}:(\mathrm{X}, \tau) \rightarrow(\mathrm{Y}, \sigma)$ is an intuitionistic fuzzy $\operatorname{rg} \alpha$ closed mapping (IFRG $\alpha$ CM for short) if image of every IFCS of $\mathrm{X}$ is an IFRG $\alpha$ CS in Y.

\subsection{Definition [2]}


Let $\mathrm{f}:(\mathrm{X}, \tau) \rightarrow(\mathrm{Y}, \sigma)$ be a mapping. Then $\mathrm{f}$ is said to be intuitionistic fuzzy homeomorphism (IF homeomorphism for short) if $\mathrm{f}$ and $f^{-1}$ are IF continuous mappings.

\subsection{Definition [10]}

Let $\mathrm{f}:(\mathrm{X}, \tau) \rightarrow(\mathrm{Y}, \sigma)$ be a mapping. Then $\mathrm{f}$ is said to be intuitionistic fuzzy $\alpha$ homeomorphism (IF $\alpha$ homeomorphism for short) if $\mathrm{f}$ and $f^{-1}$ are IF $\alpha$ continuous mappings.

\section{INTUITIONISTIC FUZZY pgr $\alpha$ CLOSED MAPPING AND INTUITIONISTIC FUZZY pgr $\alpha$ OPEN MAPPING}

In this section we introduce intuitionistic fuzzy pgr $\alpha$ closed mapping, intuitionistic fuzzy $\operatorname{pgr} \alpha$ open mapping and investigate some of its properties.

\subsection{Definition}

A mapping $\mathrm{f:}(\mathrm{X}, \tau) \rightarrow(\mathrm{Y}, \sigma)$ is called an intuitionistic fuzzy pgr $\alpha$ closed mapping (IF pgr $\alpha \mathrm{CM}$ for short) if $\mathrm{f}(\mathrm{A})$ is an IF pgr $\alpha$ CS in Y for each IFCS A in X.

\subsection{Example}

Let $\mathrm{X}=\{\mathrm{a}, \mathrm{b}\}, \mathrm{Y}=\{\mathrm{u}, \mathrm{v}\}$ and $\mathrm{G}_{1}=\langle\mathrm{x},(0.8,0.7),(0.2,0.2)\rangle$, $\mathrm{G}_{2}=<\mathrm{x},(0.3,0.3),(0.7,0.7)>$. Then $\tau=\left\{0_{\sim}, \mathrm{G}_{1}, 1_{\sim}\right\}$ and $\sigma$ $=\left\{0_{\sim}, \mathrm{G}_{2}, 1_{\sim}\right\}$ are IFTs on $\mathrm{X}$ and $\mathrm{Y}$ respectively. Define a mapping $\mathrm{f}:(\mathrm{X}, \tau) \rightarrow(\mathrm{Y}, \sigma)$ by $\mathrm{f}(\mathrm{a})=\mathrm{u}$ and $\mathrm{f}(\mathrm{b})=\mathrm{v}$. Then $\mathrm{f}$ is an IF pgr $\alpha$ CM.

\subsection{Definition}

A mapping $\mathrm{f:}(\mathrm{X}, \tau) \rightarrow(\mathrm{Y}, \sigma)$ is called an intuitionistic fuzzy pgr $\alpha$ open mapping (IF pgr $\alpha$ OM for short) if $\mathrm{f}(\mathrm{A})$ is an IF pgr $\alpha$ OS in Y for each IFOS A in X.

\subsection{Definition}

A mapping $\mathrm{f:}(\mathrm{X}, \tau) \rightarrow(\mathrm{Y}, \sigma)$ is called an intuitionistic fuzzy ipgr $\alpha$ closed mapping (IFipgr $\alpha \mathrm{CM}$ for short) if $\mathrm{f}(\mathrm{A})$ is an IF pgr $\alpha$ CS in Y for each IF pgr $\alpha$ CS A in X.

\subsection{Example}

Let $\mathrm{X}=\{\mathrm{a}, \mathrm{b}\}, \quad \mathrm{Y}=\{\mathrm{u}, \mathrm{v}\}$ and $\mathrm{G}_{1}=\langle\mathrm{x},(0.3,0.3),(0.7,0.7)\rangle$, $\mathrm{G}_{2}=<\mathrm{x},(0.8,0.7),(0.2,0.2)>$. Then $\tau=\left\{0_{\sim}, \mathrm{G}_{1}, 1_{\sim}\right\}$ and $\sigma$ $=\left\{0_{\sim}, \mathrm{G}_{2}, 1_{\sim}\right\}$ are IFTs on $\mathrm{X}$ and $\mathrm{Y}$ respectively. Define a mapping $\mathrm{f}:(\mathrm{X}, \tau) \rightarrow(\mathrm{Y}, \sigma)$ by $\mathrm{f}(\mathrm{a})=\mathrm{u}$ and $\mathrm{f}(\mathrm{b})=\mathrm{v}$. Then $\mathrm{f}$ is an IFipgr $\alpha$ CM.

\subsection{Definition}

A mapping $\mathrm{f}:(\mathrm{X}, \tau) \rightarrow(\mathrm{Y}, \sigma)$ is called an intuitionistic fuzzy ipgr $\alpha$ open mapping (IFipgr $\alpha$ OM for short) if $\mathrm{f}(\mathrm{A})$ is an IF pgra OS in Y for each IF pgr $\alpha$ OS A in X.

\subsection{Definition}

Let $(\mathrm{X}, \tau)$ be an IFTS and $\mathrm{A}=\left\langle x, \mu_{A}, v_{A}\right\rangle$ be an IFS in $\mathrm{X}$. Then pgr $\alpha$-interior of A (pgr $\alpha$ int(A) for short) and pgr $\alpha$ closure of A (pgr $\alpha \mathrm{cl}(\mathrm{A})$ for short) are defined as
1. $\operatorname{pgr} \alpha \operatorname{int}(\mathrm{A})=\cup\{G: G$ is an IF pgr $\alpha$ OS in $\mathrm{X}$ and $\mathrm{G} \subseteq A\}$,

2. $\operatorname{pgr} \alpha \operatorname{cl}(\mathrm{A})=\cap\{\mathrm{K}: \mathrm{K}$ is an IF pgr $\alpha \mathrm{CS}$ in $\mathrm{X}$ and $\mathrm{A} \subseteq K\}$.

\subsection{Theorem}

Every IFCM is an IF pgr $\alpha$ CM but not conversely.

\section{Proof:}

Let $\mathrm{f}:(\mathrm{X}, \tau) \rightarrow(\mathrm{Y}, \sigma)$ be an IF closed mapping. Let $\mathrm{A}$ be an IFCS in $\mathrm{X}$. Then $\mathrm{f}(\mathrm{A})$ is IFCS in $\mathrm{Y}$. This implies that $\mathrm{f}(\mathrm{A})$ is an IF pgr $\alpha$ CS inY. Hence $\mathrm{f}$ is an IF pgr $\alpha$ closed mapping.

\subsection{Example}

In Example 3.2, $\mathrm{f:}(\mathrm{X}, \tau) \rightarrow(\mathrm{Y}, \sigma)$ is an IF pgr $\alpha \mathrm{CM}$ but not an IFCM.

\subsection{Theorem}

Every IF $\alpha \mathrm{CM}$ is an IF pgr $\alpha \mathrm{CM}$ but not conversely.

\section{Proof:}

Let $\mathrm{f}:(\mathrm{X}, \tau) \rightarrow(\mathrm{Y}, \sigma)$ be an IF $\alpha$ closed mapping. Let $\mathrm{A}$ be an IFCS in X. Then $\mathrm{f}(\mathrm{A})$ is IF $\alpha$ CS in Y. This implies that $\mathrm{f}(\mathrm{A})$ is an IF pgr $\alpha$ CS in Y. Hence $\mathrm{f}$ is an IF pgr $\alpha$ closed mapping.

\subsection{Example}

In Example 3.2, $\mathrm{f}:(\mathrm{X}, \tau) \rightarrow(\mathrm{Y}, \sigma)$ is an IF pgr $\alpha \mathrm{CM}$ but not an IF $\alpha \mathrm{CM}$.

\subsection{Theorem}

Every IFPCM is an IF pgr $\alpha$ CM but not conversely.

\section{Proof:}

Let $\mathrm{f}:(\mathrm{X}, \tau) \rightarrow(\mathrm{Y}, \sigma)$ be an IFP closed mapping. Let $\mathrm{A}$ be an IFCS in X. Then $\mathrm{f}(\mathrm{A})$ is IFPCS in Y. This implies that $\mathrm{f}(\mathrm{A})$ is an IF pgr $\alpha \mathrm{CS}$ in Y. Hence $\mathrm{f}$ is an IF pgr $\alpha$ closed mapping.

\subsection{Example}

Let $\mathrm{X}=\{\mathrm{a}, \mathrm{b}\}, \quad \mathrm{Y}=\{\mathrm{u}, \mathrm{v}\}$ and $\mathrm{G}_{1}=\langle\mathrm{x},(0.3,0.1),(0.7,0.9)\rangle$, $\mathrm{G}_{2}=<\mathrm{x},(0.7,0.9),(0.3,0.1)>$. Then $\tau=\left\{0_{\sim}, \mathrm{G}_{1}, 1_{\sim}\right\}$ and $\sigma$ $=\left\{0_{\sim}, \mathrm{G}_{2}, 1_{\sim}\right\}$ are IFTs on $\mathrm{X}$ and $\mathrm{Y}$ respectively. Define a mapping $\mathrm{f}:(\mathrm{X}, \tau) \rightarrow(\mathrm{Y}, \sigma)$ by $\mathrm{f}(\mathrm{a})=\mathrm{u}$ and $\mathrm{f}(\mathrm{b})=\mathrm{v}$. Then $\mathrm{f}$ is an IF pgr $\alpha$ CM but not IFPCM.

\subsection{Theorem}

Every IFRG $\alpha \mathrm{CM}$ is an IF pgr $\alpha \mathrm{CM}$ but not conversely.

\section{Proof:}

Let $\mathrm{f}:(\mathrm{X}, \tau) \rightarrow(\mathrm{Y}, \sigma)$ be an IFRG $\alpha \mathrm{CM}$. Let A be an IFCS in $\mathrm{X}$. Then $\mathrm{f}(\mathrm{A})$ is IFRG $\alpha$ CS in Y. This implies that $\mathrm{f}(\mathrm{A})$ is an IF pgr $\alpha \mathrm{CS}$ in $\mathrm{Y}$. Hence $\mathrm{f}$ is an IF $\operatorname{pgr} \alpha$ closed mapping. 


\subsection{Example}

In Example 3.2, $\mathrm{f:}(\mathrm{X}, \tau) \rightarrow(\mathrm{Y}, \sigma)$ is an IF pgr $\alpha \mathrm{CM}$ but not an IFRG $\alpha$ CM.

\subsection{Theorem}

Every IF ipgr $\alpha \mathrm{CM}$ is an IF pgr $\alpha \mathrm{CM}$ but not conversely.

\section{Proof:}

Let $\mathrm{f}:(\mathrm{X}, \tau) \rightarrow(\mathrm{Y}, \sigma)$ be an IF ipgr $\alpha \mathrm{CM}$. Let A be an IFCS in X. Then A is IF pgr $\alpha$ CS in Y. This implies that $\mathrm{f}(\mathrm{A})$ is an IF pgr $\alpha$ CS in Y. Hence $\mathrm{f}$ is an IF pgr $\alpha$ closed mapping.

\subsection{Example}

Let $\mathrm{X}=\{\mathrm{a}, \mathrm{b}\}, \mathrm{Y}=\{\mathrm{u}, \mathrm{v}\}$ and $\mathrm{G}_{1}=\langle\mathrm{x},(0.8,0.7),(0.2,0.2)\rangle$, $\mathrm{G}_{2}=<\mathrm{x},(0.3,0.3),(0.7,0.7)>$. Then $\tau=\left\{0_{\sim}, \mathrm{G}_{1}, 1_{\sim}\right\}$ and $\sigma$ $=\left\{0_{\sim}, \mathrm{G}_{2}, 1_{\sim}\right\}$ are IFTs on $\mathrm{X}$ and $\mathrm{Y}$ respectively. Define a mapping $\mathrm{f}:(\mathrm{X}, \tau) \rightarrow(\mathrm{Y}, \sigma)$ by $\mathrm{f}(\mathrm{a})=\mathrm{u}$ and $\mathrm{f}(\mathrm{b})=\mathrm{v}$. Then $\mathrm{f}$ is an IF pgr $\alpha \mathrm{CM}$ but not IF ipgr $\alpha \mathrm{CM}$.

\subsection{Theorem}

A mapping f: $(\mathrm{X}, \tau) \rightarrow(\mathrm{Y}, \sigma)$ is IF pgr $\alpha$ closed mapping if and only if for each subset $\mathrm{S}$ of $\mathrm{Y}$ and for each IFOS $\mathrm{U}$ containing $f^{-1}(\mathrm{~S})$ there is an IF pgra OS V of Y such that $\mathrm{S} \subseteq \mathrm{V}$ and $f^{-1}(\mathrm{~V}) \subseteq \mathrm{U}$.

\section{Proof:}

Suppose $\mathrm{f}$ is an IF pgr $\alpha$ closed. Let $\mathrm{S}$ be a subset of $\mathrm{Y}$ and $\mathrm{U}$ is an IFOS of $\mathrm{X}$ such that $f^{-1}(\mathrm{~S}) \subseteq \mathrm{U}$. Then $\mathrm{V}=$ $\mathrm{Y}-\mathrm{f}(\mathrm{X}-\mathrm{U})$ is an IF pgr $\alpha$ OS containing $\mathrm{S}$ such that $f^{-1}(\mathrm{~V}) \subseteq \mathrm{U}$.

Conversely, suppose that $\mathrm{F}$ is an IFCS in $\mathrm{X}$. Then $f^{-1}(\mathrm{Y}-\mathrm{f}(\mathrm{F})) \subseteq \mathrm{X}-\mathrm{F}, \mathrm{X}-\mathrm{F}$ is an IFOS in $\mathrm{X}$. By hypothesis, there is an IF pgr $\alpha$ OS V of Y such that $\mathrm{Y}-\mathrm{f}(\mathrm{F}) \subseteq \mathrm{V}$ and $f^{-1}(\mathrm{~V}) \subseteq \mathrm{X}$-F.Therefore $\mathrm{F} \subseteq \mathrm{X}-f^{-1}(\mathrm{~V})$. Hence $\mathrm{Y}-\mathrm{V} \subseteq \mathrm{f}(\mathrm{F})$ $\subseteq \mathrm{f}\left(\mathrm{X}-f^{-1}(\mathrm{~V})\right) \subseteq \mathrm{Y}-\mathrm{V}$, which impiles $\mathrm{f}(\mathrm{F})=\mathrm{Y}-\mathrm{V}$. Since $\mathrm{Y}-\mathrm{V}$ is an IF pgr $\alpha \mathrm{CS}$ in $\mathrm{Y}, \mathrm{f}(\mathrm{F})$ is an IF pgr $\alpha \mathrm{CS}$ in $\mathrm{Y}$ and therefore $\mathrm{f}$ is an IF pgr $\alpha$ closed mapping.

\subsection{Theorem}

If $\mathrm{f:}(\mathrm{X}, \tau) \rightarrow(\mathrm{Y}, \sigma)$ is an IF pgr $\alpha \mathrm{CM}$ and $\mathrm{A}$ is an IFCS of $\mathrm{X}$, then $f_{A}: \mathrm{A} \rightarrow \mathrm{Y}$ is IF pgr $\alpha \mathrm{CM}$.

\section{Proof:}

Let $\mathrm{B} \subseteq \mathrm{A}$ be ab IFCS in A, then B is an IFCS in X. Since A is an IFCS in $\mathrm{X}, \mathrm{f}(\mathrm{B})$ is an IF pgr $\alpha \mathrm{CS}$ in $\mathrm{Y}$ as $\mathrm{f}$ is IF pgr $\alpha$ CM. But $\mathrm{f}(\mathrm{B})=f_{A}(\mathrm{~B})$. So $f_{A}(\mathrm{~B})$ is an IF pgr $\alpha$ CS in Y. Therefore $f_{A}$ is an IF pgr $\alpha$ CM.

\subsection{Remark}

Composition of two IF pgr $\alpha$ CMs need not be an IF pgr $\alpha$ CM.

\subsection{Example}

Let $\mathrm{X}=\{\mathrm{a}, \mathrm{b}\}, \quad \mathrm{Y}=\{\mathrm{c}, \mathrm{d}\} \quad$ and $\mathrm{Z}=\{\mathrm{u}, \mathrm{v}\}$. Let $\mathrm{G}_{1}=\langle\mathrm{x},(0.5,0.6),(0.5,0.4)\rangle, \mathrm{G}_{2}=\langle\mathrm{x},(0.6,0.1),(0.4,0.3)\rangle$ and $\mathrm{G}_{3}=<\mathrm{x},(0.4,0.4),(0.6,0.6)>$, Then $\tau=\left\{0_{\sim}, \mathrm{G}_{1}, 1_{\sim}\right\}, \sigma=\left\{0_{\sim}\right.$, $\left.\mathrm{G}_{2}, 1_{\sim}\right\}$ and $\gamma=\left\{0_{\sim}, \mathrm{G}_{3}, 1_{\sim}\right\}$ are IFTs on $\mathrm{X}$ and $\mathrm{Y}$ respectively. Define a mapping $\mathrm{f}:(\mathrm{X}, \tau) \rightarrow(\mathrm{Y}, \sigma)$ by $\mathrm{f}(\mathrm{a})=\mathrm{c}$ and $\mathrm{f}(\mathrm{b})=\mathrm{d}$ and $\mathrm{g}:(\mathrm{Y}, \sigma) \rightarrow(\mathrm{Z}, \gamma)$ by $\mathrm{g}(\mathrm{c})=\mathrm{u}$ and $\mathrm{g}(\mathrm{d})=\mathrm{v}$. Then $\mathrm{f}$ and $\mathrm{g}$ are an IF pgr $\alpha \mathrm{CM}$. But their composition gof: $(\mathrm{X}, \tau) \rightarrow(\mathrm{Z}, \gamma)$ need not be an IF pgr $\alpha \mathrm{CM}$.

\subsection{Theorem}

If $\mathrm{f:}(\mathrm{X}, \tau) \rightarrow(\mathrm{Y}, \sigma)$ is an IFCM and $\mathrm{g}:(\mathrm{Y}, \sigma) \rightarrow(\mathrm{Z}, \gamma)$ is an IF pgr $\alpha \mathrm{CM}$, then gof: $(\mathrm{X}, \tau) \rightarrow(\mathrm{Z}, \gamma)$ is an IF pgr $\alpha \mathrm{CM}$.

\section{Proof:}

Let $\mathrm{H}$ be an IFCS in $\mathrm{X}$. Then $\mathrm{f}(\mathrm{H})$ is an IFCS. But $(\mathrm{gof})(\mathrm{H})=\mathrm{g}(\mathrm{f}(\mathrm{H}))$ is an IF pgr $\alpha \mathrm{CS}$ as $\mathrm{g}$ ia an IF pgr $\alpha \mathrm{CM}$. Thus gof is an IF pgr $\alpha$ CM.

\subsection{Theorem}

If $\mathrm{f:}(\mathrm{X}, \tau) \rightarrow(\mathrm{Y}, \sigma)$ is a bijective mapping, then the following statements are equivalent

1. $\mathrm{f}$ is an IF pgr $\alpha$ OM.

2. $\mathrm{f}$ ia an IF pgr $\alpha \mathrm{CM}$.

3. $f^{-1}:(\mathrm{Y}, \sigma) \rightarrow(\mathrm{X}, \tau)$ is an IF pgr $\alpha$ continuous.

\section{Proof:}

$(1) \Rightarrow(2)$ Let $\mathrm{U}$ be an IFCS in $\mathrm{X}$ and $\mathrm{f}$ be an IF pgr $\alpha$ OM. Then $X-U$ is an IFOS in X. By hypothesis, we get $f(X-U)$ is an IF pgr $\alpha$ OS in $\mathrm{Y}$. That is $\mathrm{Y}-\mathrm{f}(\mathrm{X}-\mathrm{U})-\mathrm{f}(\mathrm{U})$ is an IF pgr $\alpha$ in Y.

$(2) \Rightarrow(3) \mathrm{L}$ is an et $\mathrm{U}$ ne an IFCS in X. By assumption, $\mathrm{f}(\mathrm{U})$ IF pgr $\alpha$ CS in Y. As $\mathrm{f}(\mathrm{U})=\left(f^{-1}\right)^{-1}(\mathrm{U}), f^{-1}$ is an IF pgr $\alpha$ continuous.

(3) $\Rightarrow(1)$ Let $U$ be an IFOS in $X$. By assumption $\left(f^{-1}\right)^{-1}(\mathrm{U})=\mathrm{f}(\mathrm{U})$. That is $\mathrm{f}(\mathrm{U})$ is an IF pgr $\alpha$ OS in $\mathrm{Y}$. Hence $\mathrm{f}$ is an IF pgr $\alpha$ OM.

\subsection{Definition}

A space $(\mathrm{X}, \tau)$ is called an IFpgr $\alpha T_{1 / 2}$ space if every IF $\operatorname{pgr} \alpha \mathrm{CS}$ is an IF $\alpha \mathrm{CS}$.

\subsection{Definition}

A space $(X, \tau)$ is called an IFpgrT $T_{1 / 2}$ space if every IF pgr $\alpha$ $\mathrm{CS}$ is an IFCS.

\subsection{Definition}

A mapping $\mathrm{f}:(\mathrm{X}, \tau) \rightarrow(\mathrm{Y}, \sigma)$ is called an intuitionistic fuzzy pgr $\alpha$ irresolute (IF pgr $\alpha$ irresolute in short) mappings if $f^{-1}(\mathrm{~V})$ is an IF $\operatorname{pgr} \alpha \mathrm{CS}$ in $(\mathrm{X}, \tau)$ for every IF pgr $\alpha$ CS $\mathrm{V}$ of $(\mathrm{Y}, \sigma)$.

\subsection{Theorem}

For any bijective mapping $\mathrm{f}:(\mathrm{X}, \tau) \rightarrow(\mathrm{Y}, \sigma)$, then the following are equivalent

1. $f^{-1}:(\mathrm{Y}, \sigma) \rightarrow(\mathrm{X}, \tau)$ is an IF pgr $\alpha$ irresolute mapping

2. $\mathrm{f}$ is an IF ipgr $\alpha \mathrm{OM}$

3. $\mathrm{f}$ is an IF ipgr $\alpha \mathrm{CM}$ 


\section{Proof:}

$(1) \Rightarrow(2)$ Let $U$ be an IF pgr $\alpha$ OS in X. By $(1),\left(f^{-1}\right)^{-1}(\mathrm{U})$ $=\mathrm{f}(\mathrm{U})$ is an IF pgr $\alpha$ OS in Y So $\mathrm{f}$ is an IF ipgr $\alpha$ OM.

$(2) \Rightarrow(3)$ Let $\mathrm{V}$ be an IF pgr $\alpha \mathrm{CS}$ in $\mathrm{X}$. By (2), $\mathrm{f}(\mathrm{X}-\mathrm{V})=$ $\mathrm{Y}-\mathrm{f}(\mathrm{V})$ is an IF pgr $\alpha$ OS in Y. That is $\mathrm{f}(\mathrm{V})$ is an IF pgr $\alpha$ CS in $\mathrm{Y}$ and so $\mathrm{f}$ is an IF ipgr $\alpha \mathrm{CM}$.

(3) $\Rightarrow$ (1) Let $\mathrm{V}$ be an IF pgr $\alpha \mathrm{CS}$ in X. By (3), $\mathrm{f}(\mathrm{V})=\left(f^{-1}\right)^{-1}(\mathrm{~V})$ is an IF pgr $\alpha$ CS in Y. Hence (1) holds.

\subsection{Theorem}

If $\mathrm{f:}(\mathrm{X}, \tau) \rightarrow(\mathrm{Y}, \sigma)$ and $\mathrm{g}:(\mathrm{Y}, \sigma) \rightarrow(\mathrm{Z}, \gamma)$ are IF ipgr $\alpha \mathrm{CM}$, then gof: $(\mathrm{X}, \tau) \rightarrow(\mathrm{Z}, \gamma)$ is an IF ipgr $\alpha \mathrm{CM}$.

\section{Proof:}

Let $\mathrm{V}$ be an IF pgr $\alpha \mathrm{CS}$ in $\mathrm{X}$. Since $\mathrm{f}$ is an IF ipgr $\alpha \mathrm{CM}$, $\mathrm{f}(\mathrm{V})$ is an IF pgr $\alpha$ CS in Y. Then $\mathrm{g}(\mathrm{f}(\mathrm{V}))$ is an IF pgr $\alpha$ CS in $\mathrm{Z}$. Hence gof is an IF ipgr $\alpha \mathrm{CM}$.

\subsection{Theorem}

If $\mathrm{f:}(\mathrm{X}, \tau) \rightarrow(\mathrm{Y}, \sigma)$ is an IF $\operatorname{pgr} \alpha \mathrm{CM}$ and $\mathrm{g}:(\mathrm{Y}, \sigma) \rightarrow$ $(\mathrm{Z}, \gamma)$ is an IF ipgr $\alpha \mathrm{CM}$, then gof: $(\mathrm{X}, \tau) \rightarrow(\mathrm{Z}, \gamma)$ is an IF pgr $\alpha$ CM.

\section{Proof:}

Let $\mathrm{V}$ be an IFCS in X. Since $\mathrm{f}$ is an IF pgr $\alpha \mathrm{CM}, \mathrm{f}(\mathrm{V})$ is an IF pgr $\alpha$ CS in Y. Then $g(f(V))$ is an IF pgr $\alpha$ CS in Z. Hence gof is an IF pgr $\alpha$ CM.

\section{INTUITIONISTIC FUZZY pgr $\alpha$ HOMEOMORPHISM}

In this section, we introduce the concept of intuitoinstic fuzzy pgr $\alpha$ homeomorphism, intuitionistic fuzzy ipgr $\alpha$ homeomorphism and study some of their properties.

\subsection{Definition}

A bijective mapping $\mathrm{f}:(\mathrm{X}, \tau) \rightarrow(\mathrm{Y}, \sigma)$ is called an intuitionistic fuzzy pgr $\alpha$ homeomorphism (IF $\operatorname{pgr} \alpha$ homeomorphism in short) if $\mathrm{f}$ and $f^{-1}$ are IF $\operatorname{pgr} \alpha$ continuous mapping.

\subsection{Example}

Let $\mathrm{X}=\{\mathrm{a}, \mathrm{b}\}, \quad \mathrm{Y}=\{\mathrm{u}, \mathrm{v}\}$ and $\mathrm{G}_{1}=\langle\mathrm{x},(0.3,0.2),(0.6,0.7)>$, $\mathrm{G}_{2}=<\mathrm{x},(0.8,0.9),(0.2,0.1)>$. Then $\tau=\left\{0_{\sim}, \mathrm{G}_{1}, 1_{\sim}\right\}$ and $\sigma$ $=\left\{0_{\sim}, \mathrm{G}_{2}, 1_{\sim}\right\}$ are IFTs on $\mathrm{X}$ and $\mathrm{Y}$ respectively. Define a mapping $\mathrm{f}:(\mathrm{X}, \tau) \rightarrow(\mathrm{Y}, \sigma)$ by $\mathrm{f}(\mathrm{a})=\mathrm{u}$ and $\mathrm{f}(\mathrm{b})=\mathrm{v}$. Then $\mathrm{f}$ is an IF pgr $\alpha$ continuous mapping and $f^{-1}$ is also an IF pgr $\alpha$ continuous mapping. Therefore $\mathrm{f}$ is an IF pgr $\alpha$ homeomorphism.

\subsection{Theorem}

Every IF homeomorphism is an IF pgr $\alpha$ homeomorphism but not conversely.

\section{Proof:}

Www.ijcat.com
Let $\mathrm{f}:(\mathrm{X}, \tau) \rightarrow(\mathrm{Y}, \sigma)$ be an IF homeomorphism. Then $\mathrm{f}$ and $f^{-1}$ are IF continuous mapping. This implies that $\mathrm{f}$ and $f^{-1}$ are IF pgr $\alpha$ continuous mapping, that is the mapping $\mathrm{f}$ is an IF pgr $\alpha$ homeomorphism.

\subsection{Example}

Let $\mathrm{X}=\{\mathrm{a}, \mathrm{b}\}, \quad \mathrm{Y}=\{\mathrm{u}, \mathrm{v}\}$ and $\mathrm{G}_{1}=\langle\mathrm{x},(0.2,0.3),(0.8,0.7)\rangle$, $\mathrm{G}_{2}=<\mathrm{x},(0.6,0.8),(0.3,0.2)>$. Then $\tau=\left\{0_{\sim}, \mathrm{G}_{1}, 1_{\sim}\right\}$ and $\sigma$ $=\left\{0_{\sim}, \mathrm{G}_{2}, 1_{\sim}\right\}$ are IFTs on $\mathrm{X}$ and $\mathrm{Y}$ respectively. Define a mapping $\mathrm{f}:(\mathrm{X}, \tau) \rightarrow(\mathrm{Y}, \sigma)$ by $\mathrm{f}(\mathrm{a})=\mathrm{u}$ and $\mathrm{f}(\mathrm{b})=\mathrm{v}$. Then $\mathrm{f}$ is an IF pgr $\alpha$ continuous mapping and $f^{-1}$ is also an IF pgr $\alpha$ continuous mapping. Therefore $\mathrm{f}$ is an IF pgr $\alpha$ homeomorphism but not IF homeomorphism.

\subsection{Theorem}

Every IF $\alpha$ homeomorphism is an IF pgr $\alpha$ homeomorphism but not conversely.

\section{Proof:}

Let $\mathrm{f}:(\mathrm{X}, \tau) \rightarrow(\mathrm{Y}, \sigma)$ be an IF $\alpha$ homeomorphism. Then $\mathrm{f}$ and $f^{-1}$ are IF $\alpha$ continuous mapping. This implies that $\mathrm{f}$ and $f^{-1}$ are IF $\operatorname{pgr} \alpha$ continuous mapping, that is the mapping $\mathrm{f}$ is an IF pgr $\alpha$ homeomorphism.

\subsection{Example}

Let $\mathrm{X}=\{\mathrm{a}, \mathrm{b}\}, \quad \mathrm{Y}=\{\mathrm{u}, \mathrm{v}\}$ and $\mathrm{G}_{1}=\langle\mathrm{x},(0.5,0.1),(0.5,0.9)\rangle$, $\mathrm{G}_{2}=<\mathrm{x},(0.2,0.2),(0.7,0.8)>$. Then $\tau=\left\{0_{\sim}, \mathrm{G}_{1}, 1_{\sim}\right\}$ and $\sigma=$ $\left\{0_{\sim}, \mathrm{G}_{2}, 1_{\sim}\right\}$ are IFTs on $\mathrm{X}$ and $\mathrm{Y}$ respectively. Define a mapping $\mathrm{f}:(\mathrm{X}, \tau) \rightarrow(\mathrm{Y}, \sigma)$ by $\mathrm{f}(\mathrm{a})=\mathrm{u}$ and $\mathrm{f}(\mathrm{b})=\mathrm{v}$. Therefore $\mathrm{f}$ is an IF $\operatorname{pgr} \alpha$ homeomorphism. Consider the IFCS $\mathrm{A}=$ $\langle\mathrm{x},(0.7,0.8),(0.2,0.2)\rangle$ in $\mathrm{Y}$. Then $f^{-1}$ (A) = $<\mathrm{x},(0.7,0.8),(0.2,0.2)\rangle$ is not IF $\alpha \mathrm{CS}$ in $\mathrm{X}$. This implies that $\mathrm{f}$ is not an IF $\alpha$ continuous mapping. Hence $\mathrm{f}$ is not an IF $\alpha$ homeomorphism.

\subsection{Theorem}

Let $\mathrm{f}:(\mathrm{X}, \tau) \rightarrow(\mathrm{Y}, \sigma)$ be an IF pgr $\alpha$ homeomorphism, then $\mathrm{f}$ is an IF homeomorphism if $\mathrm{X}$ and $\mathrm{Y}$ are IFpgrT $T_{1 / 2}$ space.

\section{Proof:}

Let $\mathrm{B}$ be an IFCS in Y. Then $f^{-1}(\mathrm{~B})$ is an IF pgr $\alpha$ CS in $\mathrm{X}$, by hypothesis. Since $\mathrm{X}$ is an $\operatorname{IFpgr}_{1 / 2}$ space, $f^{-1}(\mathrm{~B})$ is an IFCS in $X$. Hence $f$ is an IF continuous mapping. By hypothesis $f^{-1}$ : $(\mathrm{Y}, \sigma) \rightarrow(\mathrm{X}, \tau)$ is a IF $\operatorname{pgr} \alpha$ continuous mapping. Let $\mathrm{A}$ be an IFCS in X. Then $\left(f^{-1}\right)^{-1}(\mathrm{~A})=\mathrm{f}(\mathrm{A})$ is an IF pgr $\alpha$ CS in $\mathrm{Y}$, by hypothesis. Since $\mathrm{Y}$ is an IFpgrT $_{1 / 2}$ space, $\mathrm{f}(\mathrm{A})$ is an IFCS in Y. Hence $f^{-1}$ is an IF continuous mapping. Therefore the mapping $f$ is an IF homeomorphism.

\subsection{Theorem}

Let $\mathrm{f}:(\mathrm{X}, \tau) \rightarrow(\mathrm{Y}, \sigma)$ be an IF pgr $\alpha$ homeomorphism, then $\mathrm{f}$ is an IF $\alpha$ homeomorphism if $\mathrm{X}$ and $\mathrm{Y}$ are IFpgr $\alpha T_{1 / 2}$ space.

\section{Proof:}

Let $\mathrm{B}$ be an IFCS in Y. Then $f^{-1}(\mathrm{~B})$ is an IF pgr $\alpha$ CS in $\mathrm{X}$, by hypothesis. Since $\mathrm{X}$ is an IFpgr $\alpha T_{1 / 2}$ space, $f^{-1}$ (B) 
is an IF $\alpha$ CS in $\mathrm{X}$. Hence $\mathrm{f}$ is an IF $\alpha$ continuous mapping. By hypothesis $f^{-1}:(\mathrm{Y}, \sigma) \rightarrow(\mathrm{X}, \tau)$ is a IF pgr $\alpha$ continuous mapping. Let $\mathrm{A}$ be an IFCS in $\mathrm{X}$. Then $\left(f^{-1}\right)^{-1}(\mathrm{~A})=\mathrm{f}(\mathrm{A})$ is an IF $\operatorname{pgr} \alpha \mathrm{CS}$ in $\mathrm{Y}$, by hypothesis. Since $\mathrm{Y}$ is an IFpgr $\alpha T_{1 / 2}$ space, $\mathrm{f}(\mathrm{A})$ is an IF $\alpha$ CS in Y. Hence $f^{-1}$ is an IF $\alpha$ continuous mapping. Therefore the mapping $\mathrm{f}$ is an IF $\alpha$ homeomorphism.

\subsection{Theorem}

Let $\mathrm{f}:(\mathrm{X}, \tau) \rightarrow(\mathrm{Y}, \sigma)$ be a bijective mapping. If $\mathrm{f}$ is an IF pgr $\alpha$ continuous mapping, then the following are equivalent

1.f is an IF pgr $\alpha$ CM.

2.f is an IF pgr $\alpha$ OM.

3.f is an IF pgr $\alpha$ homeomorphism.

\section{Proof:}

$(1) \Rightarrow(2)$ Let $\mathrm{f}:(\mathrm{X}, \tau) \rightarrow(\mathrm{Y}, \sigma)$ be a bijective mapping and IF pgr $\alpha$ CM. This implies that $f^{-1}:(\mathrm{Y}, \sigma) \rightarrow(\mathrm{X}, \tau)$ is an IF pgr $\alpha$ continuous mapping. That is, every IFOS in $\mathrm{X}$ is an IF pgr $\alpha$ OS in Y. Hence $f^{-1}$ is an IF pgr $\alpha$ OM.

$(2) \Rightarrow(3)$ Let $\mathrm{f}:(\mathrm{X}, \tau) \rightarrow(\mathrm{Y}, \sigma)$ be a bijective mapping and IF pgr $\alpha$ OM. This implies that $f^{-1}:(\mathrm{Y}, \sigma) \rightarrow(\mathrm{X}, \tau)$ is an IF $\operatorname{pgr} \alpha$ continuous mapping. Hence $\mathrm{f}$ and $f^{-1}$ are IF $\operatorname{pgr} \alpha$ continuous mapping. That is, $\mathrm{f}$ is an IF pgr $\alpha$ homeomorphism.

(3) $\Rightarrow$ (1) Let $\mathrm{f}$ be an IF pgr $\alpha$ homeomorphism. That is, f and $f^{-1}$ are IF pgr $\alpha$ continuous mappings. Since every IFCS in $\mathrm{X}$ is an IF pgr $\alpha \mathrm{CS}$ in $\mathrm{Y}, \mathrm{f}$ is an IF pgr $\alpha \mathrm{CM}$.

\subsection{Definition}

A bijective mapping $\mathrm{f}:(\mathrm{X}, \tau) \rightarrow(\mathrm{Y}, \sigma)$ is called an intuitionistic fuzzy ipgr $\alpha$ homeomorphism (IF ipgr $\alpha$ homeomorphism in short) if $\mathrm{f}$ and $f^{-1}$ are IF $\operatorname{pgr} \alpha$ irresolute mappings.

\subsection{Theorem}

Every IFipgr $\alpha$ homeomorphism is an IF pgr $\alpha$ homeomorphism but not conversely.

\section{Proof:}

Let $\mathrm{f}:(\mathrm{X}, \tau) \rightarrow(\mathrm{Y}, \sigma)$ be an IF ipgr $\alpha$ homeomorphism. Let $\mathrm{B}$ be IFCS in Y. This implies B is an IF pgr $\alpha$ CS in Y. By hypothesis $f^{-1}(\mathrm{~B})$ is an IF pgr $\alpha \mathrm{CS}$ in $\mathrm{X}$. Hence $\mathrm{f}$ is an IF pgr $\alpha$ continuous mapping. Similarly, we can prove $f^{-1}$ is an IF pgr $\alpha$ continuous mapping. Hence $\mathrm{f}$ and $f^{-1}$ are IF pgr $\alpha$ continuous mapping. This implies that the mapping $f$ is an IF pgr $\alpha$ homeomorphism.

\subsection{Example}

Let $X=\{a, b\}, \quad Y=\{u, v\}$ and $G_{1}=<x,(0.3,0.2),(0.7,0.8)>$, $\mathrm{G}_{2}=<\mathrm{x},(0.9,0.9),(0.1,0.1)>$. Then $\tau=\left\{0_{\sim}, \mathrm{G}_{1}, 1_{\sim}\right\}$ and $\sigma$ $=\left\{0_{\sim}, \mathrm{G}_{2}, 1_{\sim}\right\}$ are IFTs on $\mathrm{X}$ and $\mathrm{Y}$ respectively. Define a mapping $\mathrm{f}:(\mathrm{X}, \tau) \rightarrow(\mathrm{Y}, \sigma)$ by $\mathrm{f}(\mathrm{a})=\mathrm{u}$ and $\mathrm{f}(\mathrm{b})=\mathrm{v}$. Therefore $\mathrm{f}$ is an IF $\operatorname{pgr} \alpha$ homeomorphism. Consider the IFCS $\mathrm{A}=\langle\mathrm{x},(0.4,0.2),(0.6,0.8)>$ in Y. Clearly A is an IF pgr $\alpha \mathrm{CS}$. But $f^{-1}(\mathrm{~A})$ is not IF $\alpha$ CS in X. That is $\mathrm{f}$ is not an IF pgr $\alpha$ irresolute mapping. Hence $\mathrm{f}$ is not an IF ipgr $\alpha$ homeomorphism.

\subsection{Theorem}

The composition of two IF ipgr $\alpha$ homeomorphisms is IF ipgr $\alpha$ homeomorphism in general.

\section{Proof:}

Let $\mathrm{f}:(\mathrm{X}, \tau) \rightarrow(\mathrm{Y}, \sigma)$ and $\mathrm{g}:(\mathrm{Y}, \sigma) \rightarrow(\mathrm{Z}, \gamma)$ be two any IF ipgr $\alpha$ homeomorphisms. Let $\mathrm{A}$ be an IF $\operatorname{pgr} \alpha \mathrm{CS}$ in $\mathrm{Z}$. Then by hypothesis, $g^{-1}(\mathrm{~A})$ is an IF pgr $\alpha$ CS in Y. Again by hypothesis, $f^{-1}\left(g^{-1}(\mathrm{~A})\right)$ is an IF $\operatorname{pgr} \alpha \mathrm{CS}$ in $\mathrm{X}$. Therefore gof is an IF pgr $\alpha$ irresolute mapping. Now let B be an IF pgr $\alpha$ CS in $\mathrm{X}$. Then by hypothesis, $\mathrm{f}(\mathrm{B})$ is an IF pgr $\alpha$ CS in Y and also $\mathrm{g}(\mathrm{f}(\mathrm{B}))$ is an IF pgr $\alpha$ CS in Z. This implies gof is an IF pgr $\alpha$ irresolute mapping. Hence gof is an IF ipgr $\alpha$ homeomorphism.

\section{REFERENCES}

[1] K.T. Atanassov, Intuitionistic fuzzy sets, Fuzzy Sets and Systems, 20(1986), 87-96

[2] D. Coker, An introduction to intuitionistic fuzzy topological spaces, Fuzzy Sets and systems, as (1997) 8189.

[3] H. Gurcay, D. Coker and Es. A. Hayder, On fuzzy continuity in intuitionistic fuzzy topological fuzzy spaces, The Journal of Fuzzy Mathematics, 5(1997), 365-378

[4] A. Joung Kon Jeon, Young Bae Jun and Jin Han Park, Intuitionistic fuzzy alpha continuity and intuitionistic fuzzy pre continuity, International Journal of Mathematics and Mathematical Sciences, 19 (2005), 3091-31011.

[5] Jyoti Pandey Bajpal and S.S. Thakur, Intuitionistic fuzzy $\operatorname{rg} \alpha$ continuity, Int. J. Contemp, Math. Sciences, 6(2011), 2335-2351.

[6] P. Rajarajeswari and L.Senthil Kumar, Regular weakly generalized closed sets in intuitionistic fuzzy topological spaces, International Journal of computer Applications, 43(18) (2012), 13-17.

[7] P. Rajarjeswari and R.Krishna Moorthy, On Intuitionistic fuzzy weakly generalized closed set and its applications, Int. J. Comput, Appl., 27(11)(2011), 9-13

[8] P.Rajrajeswari and L. Senthil Kumar, Regular weakly generalized continuous mappings in intuitionistic fuzzy topological spaces, International journal of Mathematical Archive, 3(5) (2012), 1957-1962.

[9] K. Sakthivel, Intuitionistic fuzzy alpha generalized continuous mapping and intuitionistic fuzzy alpha generalized irresolute mappings, Applied Mathematical Sciences, 4(2010), 1831-1842.

[10] K. Sakthivel, Alpha generalized homeomorphism in intuitionistic fuzzy topological spaces, Notes on Intuitionistic Fuzzy Sets, 17(2011), 30-36. 


\section{International Journal of Computer Applications Technology and Research}

Volume 4- Issue 1, 63 - 69, 2015, ISSN:- 2319-8656

[11] R.Santhi and K. Sakthivel, Intuitionistic fuzzy generalized semi continuous mappings, Advances in Theoretical and Applied Mathematics, 59(2009), 73-82.

[12] Seok Jong Lee and Eun Pyo Lee, The Category of intuitionistic fuzzy topological spaces, Bull. Korean Math. Soc., 37(1) (2000), 63-76.

[13] S.S. Thakur and Rekha Chaturvedi, Generalized closed sets in intuitionistic fuzzy topology, The J.fuzzy Math., 16(3) (2008), 559-572.
[14] M. Thirumalaiswamy and A. Surya, On pgr $\alpha$ Closed Sets in Intuitionistic Fuzzy Topological Spaces,

[15] S. S. Thakur and Bajpai Pandey Jyoti, Intuitionistic Fuzzy r $g \alpha$ Closed Sets, International Journal of Fuzzy System and Rough System, 4(1) (2011),67-73.

[16] L.A. Zadeh, fuzzy sets, information and Control, 8(1955), 338-353. 